Cahiers $d u$ MONDE RUSSE

\section{Cahiers du monde russe}

Russie - Empire russe - Union soviétique et États indépendants

$62 / 4 \mid 2021$

Varia

Kevin M. KAIN and David GOLDFRANK, eds., Russia's Early Modern Orthodox Patriarchate

\title{
Laurent Tatarenko
}

\section{OpenEdition \\ Journals}

Édition électronique

URL : https://journals.openedition.org/monderusse/12717

DOI : 10.4000/monderusse. 12717

ISSN : $1777-5388$

Éditeur

Éditions de l'EHESS

\section{Édition imprimée}

Date de publication : 1 décembre 2021

Pagination : 667-672

ISBN : 978-2-7132-2895-7

ISSN : $1252-6576$

Référence électronique

Laurent Tatarenko, « Kevin M. KAIN and David GOLDFRANK, eds., Russia's Early Modern Orthodox Patriarchate ", Cahiers du monde russe [En ligne], 62/4 | 2021, mis en ligne le 01 décembre 2021, consulté le 03 septembre 2022. URL : http://journals.openedition.org/monderusse/12717 ; DOI https://doi.org/10.4000/monderusse. 12717

Ce document a été généré automatiquement le 3 septembre 2022.

Tous droits réservés 


\title{
Kevin M. KAIN and David GOLDFRANK, eds., Russia's Early Modern Orthodox Patriarchate
}

\author{
Laurent Tatarenko
}

\section{RÉFÉRENCE}

Kevin M. KAIN and David GOLDFRANK, eds., Russia's Early Modern Orthodox Patriarchate, vol. 1 : Foundations and Mitred Royalty, 1589-1647, vol. 2 : Apogee and Finale, 1648-1721, Washington - London : Academica Press, 2020, p. $284+291$

1 La publication dirigée par deux spécialistes de la culture religieuse de la Russie médiévale et moderne propose de réexaminer la première période du patriarcat de Moscou, depuis sa fondation en 1589 jusqu'à son abolition en 1721. Les seize contributions rassemblées en deux volumes sont issues du colloque international réuni en septembre 2013 à l'université de Leipzig. Aussi l'un des points forts de ce travail collectif est-il de repenser les évolutions sur le temps long pour réévaluer la nature et le poids des phénomènes qui ont façonné la construction et l'affirmation du pouvoir patriarcal en Russie.

2 Pour autant, les différents chapitres de l'ouvrage ne forment pas une véritable histoire du patriarcat de Moscou, mais apportent des éclairages sur des aspects bien définis, avec un regard neuf et original sur plusieurs sujets traités par l'historiographie classique. Même s'il s'agit d'un choix légitime, assumé par les éditeurs et parfaitement adapté à un public spécialiste, il obligera le lecteur moins familier de l'histoire de la Russie à se tourner vers d'autres publications pour resituer ces réflexions dans le contexte religieux et politique des différentes périodes considérées. Sans amoindrir la qualité du propos, cette approche peut sembler surprenante au vu du titre général de la publication, mais également de la volonté exprimée dans les textes qui ouvrent et concluent cette réflexion d'inscrire 
l'objet étudié dans un cadre chronologique plus large et de souligner sa place dans l'histoire européenne.

3 En effet, la première contribution rédigée par L. Steindorf, qui sert de chapitre introductif à l'ensemble, revient sur les grandes étapes de la formation des hiérarchies ecclésiastiques dans les christianismes latin et byzantin, en lien avec les aspirations à l'indépendance des différentes entités politiques au cours de l'époque médiévale (signalons une coquille p.18, où le siège de Preslav a été confondu avec celui de Tărnovo). L'auteur y voit une forme de réappropriation locale du principe impérial de la dyarchie, qui impliquait une correspondance entre le rang du souverain et celui du chef spirituel de l'Église locale. De cette manière, le cas russe illustre la perpétuation de l'héritage canonique byzantin et l'élévation de la métropole moscovite au statut de patriarcat devenait à la fois la conséquence tardive et le moyen de légitimer l'introduction du titre de tsar (ou basileus) dans la titulature d'Ivan IV et de ses successeurs.

4 Le récit des origines du patriarcat occupe également le chapitre suivant. E. Beljakova y étudie la narration forgée par l'Église officielle, en revenant sur la généalogie du Dit sur l'érection du patriarcat, inséré dans la première édition du nomocanon slavon (Kormčaja kniga) achevée en 1653. Ce texte était une version abrégée des Nouvelles sur le début du patriarcat en Russie (datées des années 1626-1627 et non de l'époque de Nikon, comme l'avaient supposé plusieurs travaux antérieurs), qui reprenaient elles-mêmes une œuvre du Xve siècle, destinée à défendre l'autocéphalie moscovite à partir des antécédents serbe et bulgare. Tout en tentant d'y éliminer les éléments antigrecs, la publication de 1653 y joignait la charte produite en 1589 par le secrétariat moscovite des Ambassades et les confirmations envoyées par les patriarches grecs en 1590 et 1619, qui glorifiaient le tsar comme le premier souverain du monde orthodoxe. Paradoxalement, le résultat devenait une compilation hétérogène qui fragilisait indirectement la position du chef de l'Église russe. En effet, non seulement ce récit révélait un déséquilibre entre l'autorité du tsar et celle du patriarche, placé derrière les titulaires des quatre anciens sièges patriarcaux, mais il légitimait également la position des vieux-croyants, reconnaissant le prestige de la tradition religieuse moscovite face aux normes grecques promues par Nikon. Le seul regret face à cette étude érudite et bien argumentée concerne la bibliographie de la première partie. Les références pour l'histoire des Balkans à l'époque médiévale s'y limitent ainsi aux seules monographies de B.N. Florja et de F. Dvornik, alors que les développements sur la polémique issue du rejet du concile de Ferrare-Florence fait étonnamment l'économie de citer les travaux de Ja.S. Lur'e. Plus étrangement encore, l'auteur évite de mentionner son propre livre, publié en 2017 avec L.V. Moškova et T.A. Oparina, qui présente une analyse textuelle détaillée et une édition critique du Dit sur l'érection du patriarcat.

5 Après cette seconde étude transversale, les autres textes se répartissent entre différents axes thématiques qui se développent de manière chronologique entre les deux volumes. Une place particulière y revient au problème de la nature de l'autorité patriarcale et de ses rapports avec le tsar, qui structure le propos de plusieurs chapitres.

6 Celui de D. Isaev réexamine les formulaires produits à l'époque de Philarète (1619-1633) et tend à étayer la lecture en faveur d'une « co-souveraineté » entre le tsar et le patriarche, puisque, contrairement au premier, celui-ci ne pouvait agir indépendamment dans les affaires non ecclésiastiques. L'auteur souligne, en outre, que la position singulière de Philarète était la conséquence de son lien de parenté avec le souverain. De fait, le régime 
patrimonial traditionnel, qui s'appliquait également au tsar et à sa famille, plaçait le père au-dessus du fils et l'accession au trône de Michel Romanov du vivant même de son père créait une anomalie, qui fut résolue en dotant Philarète du titre de "grand souverain ». Dès lors, le poids politique du patriarche est resté étroitement associé à son statut personnel et n'a pas permis pas de renforcer l'autorité de ses successeurs.

7 À son tour, G.B. Michels aborde la position de Philarète à travers son programme de réformes disciplinaires, énoncé au concile de 1620 et censé empêcher l'influence des " hérésies latines» en territoire moscovite. Ces mesures prévoyaient notamment de soumettre à une enquête détaillée les transfuges venus du territoire polono-lituanien et de rebaptiser par triple immersion les catholiques nouvellement convertis à l'orthodoxie. Si l'auteur voit dans ce raidissement confessionnel la volonté de protéger l'État moscovite, empêchant le retour d'un souverain polonais non orthodoxe, il remarque aussi que le patriarche se montrait plus soucieux de faire des annonces marquantes que de mener un véritable travail de fond pour mieux contrôler la discipline religieuse des nombreuses personnes qui revenaient ou fuyaient vers la Russie depuis la République polono-lituanienne. Donc, la position de Philarète visait avant tout à légitimer sa position, faisant oublier les débuts de sa carrière ecclésiastique aux côtés de Faux Dimitri et sa proximité avec les Polonais.

8 Les accents politiques des rapports entre l'Église russe et les non orthodoxes fait également l'objet du chapitre d'I. Gruber. Par une analyse minutieuse de l'attitude des autorités moscovites envers les juifs, durant la période patriarcale, l'auteur montre que la rhétorique anti-juive varia en fonction des tensions politiques internes et des conquêtes militaires des souverains moscovites. Les restrictions imposées par Ivan IV pour interdire la présence de juifs sur le territoire moscovite se sont confrontées à l'afflux de différentes populations non orthodoxes durant le Temps des Troubles, en qualité de soldats ou de marchands qui suivaient les troupes. Le même phénomène se répéta de manière encore plus massive pendant la guerre russo-polonaise des années 1654-1667, qui plaça sous l'autorité du tsar de vastes territoires avec d'importantes communautés juives et vit l'arrivée de très nombreux prisonniers de guerre. Les tsars et les patriarches tentèrent de faire face à cette situation en imposant les conversions, mais elles restaient des actes formels, comme l'atteste à la fin du xvII e siècle les usages culturels de plusieurs convertis juifs de l'entourage même des tsars, dont certains continuaient à signer les documents en caractères hébreux.

9 Tout en se concentrant sur les actions des zélateurs de la piété dans le mouvement de réforme politique et religieuse, menée à la fin des années 1640, A. Lavrov entame la série d'études consacrées à la figure du patriarche Nikon. De fait, malgré le rejet de leurs demandes au concile ecclésiastique de 1649, les zélateurs surent profiter de leur proximité avec le tsar pour placer l'un des leurs - Nikon - à la tête de la vaste métropole de Novgorod. Ce territoire devint alors un véritable laboratoire des réformes introduites par le nouveau hiérarque dans la vie religieuse locale, en contestant indirectement l'autorité du patriarche Joseph. Paradoxalement, en succédant à son opposant en 1652, Nikon hérita lui-même d'une charge patriarcale affaiblie par les attaques des zélateurs. La conclusion du chapitre suggère que, dans les années suivantes, la réflexion politique formulée par Nikon sur les rapports entre le tsar et le patriarche peut se comprendre comme une tentative de retrouver un statut qu'il avait lui-même participé à ébranler.

D. Goldfrank poursuit l'enquête sur la construction du pouvoir patriarcal en analysant les causes de la chute de Nikon. Il indique notamment que, dans la seconde moitié des 
années 1650, Nikon apparaissait non comme l'initiateur des décisions politiques, mais comme un collaborateur exalté de la monarchie engagée dans la guerre, poussant le tsar à poursuivre ses conquêtes. D'ailleurs, l'usage du titre de "grand souverain " par le patriarche - mis en exergue par ses détracteurs - lui aurait été accordé par le tsar luimême afin de renforcer son autorité pour l'application de réformes, mais également affirmer son pouvoir spirituel sur les nouveaux territoires à conquérir dans la guerre contre les Polonais. Par conséquent, le chapitre formule l'hypothèse que la déchéance du patriarche fut précipitée non par sa concurrence avec le tsar, mais par l'échec de la politique ukrainienne, qui l'impliquait directement, et par ses conflits personnels avec les conseillers laïcs du souverain.

11 Cette analyse politique s'achève avec le texte de D. Ostrowski qui réexamine les causes de l'abolition du patriarcat en 1721. Contrairement à l'idée traditionnelle qui interprète cet événement comme la conséquence des tensions entre les différents groupes du clergé, l'auteur rappelle, à la suite de P. Bushkovitch, que Pierre $\mathrm{I}^{\mathrm{er}}$ était beaucoup plus préoccupé par les opposants à son propre pouvoir et qu'il voulait éviter que la figure du patriarche devienne un symbole et un moyen de légitimation aux mains de ses ennemis. D'autre part, cette analyse affirme que la création du saint-synode était moins une rupture qu'une poursuite sous une forme nouvelle des rapports antérieurs entre l'Église et le tsar. En particulier, le programme de réformes culturelles et disciplinaires énoncé par une partie de l'élite ecclésiastique à la fin $\mathrm{du}_{\mathrm{XVII}}^{\mathrm{e}}$ siècle se poursuivit au siècle suivant, en s'accommodant de la nouvelle organisation institutionnelle, jugée par certains hiérarques plus efficace que les derniers patriarches.

Pour resituer les défis rencontrés par le patriarcat de Moscou dans un contexte ecclésiologique plus large, le livre prête également attention aux contacts entre les hiérarques russes et les différents acteurs issus des autres territoires du monde orthodoxe. Sur ce point, la contribution de N. Pissis montre que la hiérarchie grecque refusa, durant toute la période patriarcale, de reconnaître au siège de Moscou un rang similaire à celui des quatre anciens patriarcats orientaux. D'ailleurs, en renversant l'image de la corruption de la foi grecque après la conquête ottomane, les patriarches orientaux, à l'image de Dosithée de Jérusalem, n'hésitaient pas à parler de la décadence de l'Église russe en raison de l'introduction de nombreuses pratiques locales et lui déniaient une véritable autorité en matière de théologie. Par conséquent, sans contester la validité canonique de la promotion de 1589, le clergé grec continuait à considérer cet épisode comme une concession faite au tsar pour gagner son soutien et, plus largement, réinstaurer la communion entre les différentes parties de la chrétienté orientale. Le même imaginaire explique la reconnaissance rapide par Jérémie III de Constantinople de l'abolition du patriarcat de Moscou par Pierre $\mathrm{I}^{\mathrm{er}}$.

13 L'étude biographique proposée par V. Tchentsova et consacrée à la carrière d'Arsénios le Grec, proche collaborateur de Nikon, montre que le prestige revendiqué par les élites grecques était implicitement reconnu par une partie de la hiérarchie ecclésiastique moscovite. Toutefois, cette admiration pour les savants hellénophones ouvrait la voie à des individus aux parcours parfois sinueux qui suscitaient des critiques de la part de leurs compatriotes et jouaient, de ce fait, un rôle ambivalent. Si Arsénios et d'autres savants Grecs proches de Nikon, furent des agents de premier plan dans la mise en pratique de son programme de réformes cultuelles, leurs identités confessionnelles fluctuantes alimentèrent les critiques contre le patriarche. 
14 À son tour, le texte d'o. Olar dévoile les modes de circulation des idées réformatrices au sein des communautés orthodoxes à l'époque de Nikon. L'exemple des débats autour du synode de Târgovişte, convoqué en 1659 par le prince de Valachie Mihnea III Radu, montre que les problématiques ecclésiologiques abordées par Nikon et ses échanges avec Constantinople suscitaient de l'intérêt au-delà des frontières moscovites et servaient même de point de référence pour les réformes religieuses menées dans les principautés danubiennes.

Dans cette série de regards extérieurs, le premier volume comporte également le texte d'A. Brüning consacré au métropolite de Kiev Piotr Mohyła (Movilă), dont la carrière est décrite par l'auteur comme une illustration du maintien de l'imaginaire byzantin au sein du clergé de tradition slave orthodoxe de la période moderne. Malheureusement, la qualité du chapitre, qui présente une relecture originale et bien documentée du parcours de l'un des plus éminents acteurs de l'orthodoxie au XVII siècle, ne suffit pas à masquer son décalage par rapport à la problématique centrale du livre. Même s'il est incontestable que les conséquences des réformes menées par Mohyła à Kiev eurent un fort impact sur le développement de l'Église moscovite, la narration proposée dans ce chapitre se borne à la perspective ruthène et exclut de fait les rapports avec le patriarcat de Moscou (mentionné une seule fois !). Cela est d'autant plus regrettable que l'expertise de l'auteur - qui est l'un des meilleurs spécialistes du sujet - et la vaste bibliographie consacrée à Mohyła aurait pu donner lieu à différentes analyses d'histoire comparée, montrant les circulations des imaginaires religieux entre les deux parties de l'ancienne métropole de Kiev.

16 Une telle perspective apparaît justement dans un autre groupe de chapitres qui suivent l'introduction de nouveaux programmes religieux dans le domaine de l'art religieux. Celui de L.M. Evseeva observe la transformation des iconostases à l'époque du tsar Alexis et du patriarche Nikon, qui ont gagné en taille pour introduire la représentation des douze apôtres et rééquilibrer le format des différentes icônes pour affirmer la continuité entre l'Ancien et le Nouveau Testament, révélant l'économie du salut. Le passage à ce nouveau programme iconographique, plus proche du modèle grec, s'est fait par l'intermédiaire des églises ruthènes, et spécialement du monastère des Grottes de Kiev, qui a servi de référence aux églises de Moscou.

17 L'analyse d'A. Lidov propose une approche semblable en s'intéressant à la création des espaces sacrés (hiérotopie) et aux innovations artistiques de Nikon. Il montre comment le patriarche réformateur a voulu transposer en Russie les lieux saints de la Palestine du Nouveau Testament pour en faire de nouveaux pôles spirituels de la Rus'. La construction de cette topographie s'appuyait sur les nouvelles fondations monastiques et, tout d'abord, sur le complexe architectural de la Nouvelle Jérusalem, qui reprenait les traits et le langage symbolique du Saint-Sépulcre à Jérusalem. Ainsi, cette démarche visait à y produire "une icône spatiale de la Terre sainte", par une révision originale des traditions de l'art sacré byzantin.

18 La force des innovations iconographiques à l'époque de Nikon est également mise en lumière par la contribution finale de K.M. Kain qui examine le destin de son célèbre portrait avec le clergé, peint de son vivant et présenté au XIX siècle « comme le seul vrai visage de l'épiscopat russe » de cette période. Redécouvert tardivement, puis classé au rang de véritable antiquité à partir des années 1820 et diffusé sous forme d'adaptations imprimées, le tableau servit à promouvoir une image positive du patriarche, contre les critiques formulées par l'historien Solov'ev et les écrits des vieux-croyants. 
Enfin, une place particulière revient au chapitre de N.Chrissidis, qui est le seul à concentrer son propos sur la seconde moitié du XVII siècle à travers l'analyse des pratiques de charité sous le patriarcat de Joasaph II. Grâce à l'étude des registres des dépenses du Trésor patriarcal pour l'année 1671-1672, l'auteur remet en cause l'idée classique qui prétendait que l'organisation de la charité moscovite d'avant la période pétrovienne n'était que très faiblement institutionnalisée et laissait une large place aux actions informelles. La source révèle en effet une comptabilité bien structurée d'après une typologie très précise, qui associait des distributions impersonnelles à caractère " ostentatoire » avec l'aide fournie à des individus particuliers, qui consolidait les liens de patronage entre le hiérarque et ses subordonnés. Ce double système se déployait dans l'espace du Kremlin, qualifié par l'auteur de "citadelle de la charité", et servait à promouvoir le prestige du patriarche, en montrant sa piété, mais aussi son rôle social auprès des plus démunis.

La place quelque peu marginale occupée par ce travail à la fois original et novateur devient alors le symbole des rares regrets que peuvent susciter les aspects formels du livre. En effet, le choix des sujets, et contrairement au programme annoncé en introduction par les éditeurs, est très largement dominé par l'évolution de l'imaginaire politique et religieux promu par les patriarches successifs. Pourtant, il aurait été souhaitable d'élargir le propos à d'autres problématiques comme les transformations liturgiques, le personnel et le fonctionnement des institutions patriarcales, l'empreinte des réformes religieuses sur la justice ecclésiastique etc. D’ailleurs, ce déséquilibre thématique se traduit par une disparité chronologique, puisque le patriarcat de Nikon (1652-1666) concentre l'attention des deux tiers des contributions, le reste étant consacré à l'époque de Philarète (1619-1633), de Job (1589-1605) et exceptionnellement à celle de Joasaph (1667-1672), alors que les huit autres patriarcats sont à peine mentionnés. Enfin, le lecteur est parfois gêné par la disparité terminologique entre les textes, à l'exemple des populations orthodoxes de Pologne-Lituanie et de l'Hetmanat cosaque, qualifiées de « Ruthènes ", mais également d'Ukrainiens ou, de manière moins heureuse de « Russes occidentaux ».

21 Malgré ces quelques réserves, l'ouvrage constitue une étape importante dans le récent renouvellement de l'histoire religieuse de la Russie et une excellente réflexion sur l'exercice du pouvoir ecclésiastique dans le monde slave orthodoxe, vouée à devenir une référence pour tous les spécialistes du christianisme oriental.

\section{AUTEURS}

\section{LAURENT TATARENKO}

CNRS - IHMC (UMR 8066) 